\title{
ORIGINAL
}

\section{INTERVENCIÓN EDUCATIVA PARA LA PREVENCIÓN DE EMBARAZOS NO DESEADOS Y ENFERMEDADES DE TRANSMISIÓN SEXUAL EN ADOLESCENTES DE LA CIUDAD DE TOLEDO (*)}

\author{
Sonsoles Callejas Pérez (1), Beatriz Fernández Martínez (2), Paloma Méndez Muñoz (3), M. Teresa \\ León Martín (1), Carmen Fábrega Alarcón (2), Alejandro Villarín Castro (2), Óscar Rodríguez \\ Rodríguez (4), Rodrigo Bernaldo de Quirós Lorenzana (d), Ana Fortuny Tasias (1), Francisco \\ López de Castro (5) y Olga Fernández Rodríguez (5) \\ (1) Centro de Salud Santa María de Benquerencia (Toledo). \\ (2) Centro de Salud Sillería (Toledo). \\ (3) Centro de Salud de Fuensalida (Toledo). \\ (4) Centro de Salud de la Puebla de Montalbán (Toledo). \\ (5) Unidad Docente de Medicina Familiar y Comunitaria de Toledo. \\ (*) Primer Accésit para Proyectos de Actividades Comunitarias en Atención Primaria con el Proyecto «Infórmate. Así \\ serás dueño de tu futuro». PACAP-semFYC. Congreso semFYC. Barcelona, diciembre 2003. Premio a la mejor comu- \\ nicación oral «Una experiencia educativa con adolescentes». XXIV Congreso de la Sociedad Española de Medicina de \\ Familia y Comunitaria. Sevilla, diciembre 2004.
}

\section{RESUMEN}

Fundamento: Nadie duda de la necesidad de acercar de forma efectiva a los adolescentes información sobre anticoncepción y enfermedades de transmisión sexual. El objetivo de este estudio es evaluar el resultado de una intervención educativa en este ámbito.

Métodos: Estudio antes-después de una intervención educativa (basada en charlas y entrega de documentación) sin grupo control. Se pasó un cuestionario antes y después de la intervención para valorar cambios en conocimientos y actitudes a los alumnos de $4^{\circ}$ Enseñanza Secundaria Obligatoria de cinco centros educativos de Toledo.

Resultados: Contestaron 238 alumnos (de un total de 268). La edad media fue de 15,59 . El 54,66\% eran mujeres. El 24,03\% ya habían mantenido alguna relación sexual. El método anticonceptivo más utilizado fue el preservativo $(98,24 \%)$. Las chicas rechazan una relación sin protección con más frecuencia que los chicos $(76,5 \%$ vs $48,6 \%$; $<<0,001$ ) y tienen más facilidad para compartir clase con un enfermo de sida $(80,47 \%$ vs $60,38 \%$; $\mathrm{p}<0,001)$. A los seis meses del inicio de la intervención respondieron al segundo cuestionario 197 alumnos. El uso correcto del preservativo pasó del $62,13 \%$ al 73,46\%.

Conclusiones: Tras la intervención se aprecia una mejora en el nivel de conocimientos sobre anticonceptivos y sobre transmisión del sida y una actitud más positiva frente al VIH.

Palabras clave: Educación para la Salud. Salud de los Adolescentes. Embarazo en adolescencia. Enfermedades de transmission sexual.

Correspondencia:

Sonsoles Callejas Pérez.

Vía Carpetana ${ }^{\circ} 109,2^{\circ}$ dcha. 28047 Madrid.

Correo electrónico: scallejas@eresmas.com
ABSTRACT

\section{Educational Intervention for Preventing Unwanted Pregnancies and Sexually- Transmitted Diseases among Teenagers in the City of Ttoledo, Spain}

Background: No-one doubts the need of effectively providing teenagers with information about birth control and sexually-transmitted diseases. This study is aimed at evaluating the results of an educational intervention related to these matters.

Methods: Before-and-after study of an educational intervention (based on lectures and handing out documentation) without a control group. A questionnaire was passed out before and after the intervention to assess changes in knowledge and attitudes of the 4th-year Compulsory Secondary Education students at five schools in Toledo.

Results: The questionnaire was answered by 238 of the 268 students. The average age was 15.59 . A total of $54.66 \%$ were females. In all, $24.03 \%$ had had some sexual relation. The birth control method used most often was the condom (98.24\%). The girls more refuse more unprotected relations $(76.5 \%$ vs. $48.6 \%$; $<<0.001)$ and share the same classroom with a student having AIDS $(80.47 \%$ vs. $60.38 \%$; $\mathrm{p}<0.001)$. Six months following the start of the intervention, a total of 197 students answered the second questionnaire. Proper condom use rose from $62.13 \%$ to $73.46 \%$.

Conclusions: Following the intervention, an improvement has been noted in the degree of knowledge related to birth control methods and AIDS transmission and a more positive attitude regarding HIV.

Key words: Health education. Teen health. Unwanted pregnancy. Pregnancy in adolescence. Sexually transmitted diseases. 


\section{INTRODUCCIÓN}

La incidencia en España de embarazo no deseado y enfermedades de transmisión sexual (ETS) ha aumentado en los últimos años, a pesar del creciente interés social e institucional y las numerosas campañas de información general dirigidas a los jóvenes ${ }^{1-4}$.

Las interrupciones voluntarias de embarazos (IVE) van en aumento, son más frecuentes entre los 20-24 años, existiendo en la última década un incremento del doble entre las adolescentes. En la Comunidad Autónoma de Castilla-La Mancha el porcentaje de IVE en 1999 fue del 0,2\% en menores de 15 años y del 13,5\% entre los 15-19 años. En el $2000^{5}$ hubo un aumento en la demanda de anticoncepción de emergencia, siendo del 0,2\% entre los 15-19 años, el doble que en la franja de edad 20-24 años. En estadísticas más recientes de un área de salud de Madrid se recoge la cifra de $4,45 \%$ en menores de edad $^{6}$. Aunque en la última década el número de casos de sida en España ha disminuido debido al avance de los tratamientos antirretrovirales, el $32,9 \%$ se había diagnosticado en menores de 29 años. Según estadísticas del 2001 el 2,7\% de contagios en varones y el 5,9\% en mujeres entre 15-19 años fueron debidos a relaciones heterosexuales de ries$\mathrm{go}^{5}$.

Aunque ha habido experiencias educativas con resultados dispares para tratar de prevenir tanto los embarazos no deseados en adolescentes ${ }^{7-9}$ como las ETS $^{10-12}$, hay varias circunstancias que hacen que los mensajes educativos no lleguen de forma efectiva a los adolescentes: la escasa percepción del riesgo en este grupo de edad., la rebeldía hacia los mensajes procedentes de sus «mayores» y la consideración por parte de los jóvenes del preservativo como un «elemento externo» que resta espontaneidad a la relación ${ }^{13,14}$.

Los adolescentes continúan siendo una de las poblaciones donde la intervención comu- nitaria es primordial ${ }^{15}$. Las relaciones sexuales y las prácticas de riesgo para ETS, se inician cada vez antes, a partir de los 14 años, haciendo necesarios los programas de prevención en estas edades ${ }^{5,16}$. Uno de los ámbitos idóneos para el desarrollo de estas actividades preventivas son los centros educativos, ya que permiten llegar a casi todos los adolescentes y poseen importantes recursos administrativos y organizativos propios ${ }^{1}$.

Los objetivos de este trabajo son valorar los conocimientos y actitudes sobre métodos de prevención de los embarazos no deseados y las ETS y evaluar el resultado de una intervención educativa, desarrollada en el ámbito escolar, dirigida a mejorar la información sobre estos temas.

\section{SUJETOS Y MÉTODOS}

Se trata de un estudio antes-después, sin grupo control. Durante el curso escolar 2003-2004 se desarrolló una intervención educativa para la prevención de los embarazos no deseados y ETS/sida, dirigida a estudiantes de $4^{\circ}$ curso de Enseñanza Secundaria Obligatoria (ESO). Participaron cinco centros educativos de los 14 existentes en la ciudad de Toledo (a todos ellos se les visitó para ofertarles su participación), dos de titularidad privada (religiosos) y tres de titularidad pública.

La intervención se basó en la organización de un concurso de mensajes publicitarios (a modo de anuncios para prensa, radio y televisión) que debían ser elaborados por los propios estudiantes, agrupados en «talleres de salud» (de entre 5 y 8 estudiantes) y tutelados por un profesor. Cada centro docente participante fue supervisado por dos médicos residentes de Medicina Familiar y Comunitaria de tercer año, que se ocuparon de aportar material gráfico y documental, así como de impartir dos charlas educativas monográficas de unos 50 minutos de duración sobre cada uno de los temas (anticon- 
cepción y ETS). En la primera se hacía una demostración de la técnica correcta de utilización del preservativo. Para comprobar la marcha de los trabajos se mantuvo una comunicación periódica con los profesores responsables de los grupos.

Antes de la intervención se realizó un estudio descriptivo transversal mediante una encuesta anónima y autocumplimentada, construida ad hoc, que constaba de 20 preguntas sobre hábitos sexuales, utilización de métodos anticonceptivos, conocimientos y actitudes acerca de los mismos y ETS/sida. La encuesta se basó en cuestionarios previos extraídos de la bibliografía existente ${ }^{7,10,17}$. Antes de realizarla se hizo una prueba piloto con 16 adolescentes para valorar su inteligibilidad y aceptación. También se recogieron la edad y el sexo.

A los seis meses del inicio de la intervención los estudiantes respondieron otro cuestionario similar al primero, con algunas preguntas añadidas acerca de su participación y opinión sobre la utilidad de la intervención. La intención era valorar los cambios en los conocimientos y actitudes sobre la prevención de ETS y embarazos no deseados.

Los datos obtenidos de las encuestas se incluyeron en una base de datos informática del programa Rsigma. La descripción de los resultados se hizo utilizando parámetros habituales (porcentajes, medias, etc.) con los intervalos de confianza (IC 95\%) correspondientes. Para el análisis de las diferencias encontradas se emplearon el test de chi cuadrado de Pearson y el de la t de Student para comparación de porcentajes y medias respectivamente.

\section{RESULTADOS}

Aceptaron participar en la intervención cinco centros educativos de los catorce existentes en la ciudad de Toledo. Contestaron el primer cuestionario 238 alumnos de un total de 268 matriculados. La edad media fue de
15,59 (rango 14 a 18). El 54,66\% eran mujeres. El 24,03\% (IC95\% 18,80-30,14) habían mantenido en alguna ocasión relaciones sexuales con penetración, no observándose diferencias significativas en la comparación por sexos $(21,69 \%$ en hombres; $26,40 \%$ en mujeres). Sí se aprecia un menor porcentaje de adolescentes que afirmaron haber mantenido relaciones sexuales en el grupo de menores de 16 años que en el de mayor edad $(10,71 \%$ vs. $42,71 \%$; $p<0,001)$.

De entre aquellos que habían mantenido relaciones el 19,64\% lo había hecho una vez; el $42,85 \%$ de forma ocasional; el 19,64\% varias veces al mes y un $17,85 \%$ varias a la semana, no apreciándose diferencias estadísticamente significativas entre sexos. Todos habían utilizado en alguna ocasión un método anticonceptivo, siendo el más frecuente el preservativo $(98,24 \%)$. Los anticonceptivos orales habían sido empleados por el $6,06 \%$ de las chicas (edad media $17,00)$ mientras que la «píldora del día después» la habían tomado el 18,18\% de ellas (edad media 15,83). Un 12,28\% habían recurrido al menos una vez al coito interruptus. Utilizaron siempre protección en sus relaciones el $81,48 \%$ (el 90,47\% de los varones y $75,75 \%$ de mujeres; $p=0,318$ ).

Los conocimientos de los alumnos participantes sobre el preservativo se reflejan en la tabla 1. El 95,72\% saben que previene el contagio de sida y ETS y el 97,02\% que previene el embarazo. Las chicas tienen más conocimientos sobre la capacidad de prevención de embarazo y rechazan una relación sexual sin protección más frecuentemente que los chicos; por el contrario, éstos son más capaces de comprarlos y colocarlos correctamente.

Respecto a los conocimientos y actitudes frente al sida, los resultados obtenidos se encuentran en la tabla 2. Se puede apreciar cómo el porcentaje que afirma que compartiría clase con un enfermo de sida es mayor en chicas que en chicos $(80,47 \%$ vs $60,38 \%$; $p$ < 0,001). 
Tabla 1

Conocimientos y actitudes sobre los preservativos, según sexos

\begin{tabular}{|c|c|c|c|c|}
\hline \multicolumn{2}{|c|}{ Pregunta / Respuesta } & \multirow{2}{*}{$\begin{array}{c}\text { Total } \\
224(95,7 \%)\end{array}$} & \multirow{2}{*}{$\begin{array}{c}\text { Chicos } \\
101(95,2 \%)\end{array}$} & \multirow{2}{*}{$\begin{array}{c}\text { Chicas } \\
123(96,0 \%)\end{array}$} \\
\hline Previene sida/ETS & si & & & \\
\hline & no & $6(2,5 \%)$ & $3(2,8 \%)$ & $3(2,3 \%)$ \\
\hline & $\mathrm{ns} / \mathrm{nc}$ & $4(1,7 \%)$ & $2(1,8 \%)$ & $2(1,5 \%)$ \\
\hline \multirow[t]{3}{*}{ Previene embarazo } & si & $228(97,0 \%)$ & $101(94,3 \%)$ & $127(99,2 \%)$ \\
\hline & no & $6(2,5 \%)$ & $5(4,6 \%)$ & $1(0,7 \%)$ \\
\hline & $\mathrm{ns} / \mathrm{nc}$ & $1(0,4 \%)$ & $1(0,1 \%)$ & 0 \\
\hline \multirow{3}{*}{$\begin{array}{l}\text { Disminuye la sensación de } \\
\text { placer }\end{array}$} & si & $66(28,3 \%)$ & $33(31,4 \%)$ & $33(25,7 \%)$ \\
\hline & no & $73(31,3 \%)$ & $33(31,4 \%)$ & $40(31,2 \%)$ \\
\hline & $\mathrm{ns} / \mathrm{nc}$ & $94(40,3 \%)$ & $39(37,1 \%)$ & $55(42,9 \%)$ \\
\hline \multirow[t]{3}{*}{ Capaz de comprarlos } & si & $162(69,5 \%)$ & $88(83,0 \%)^{*}$ & $74(58,2 \%)^{*}$ \\
\hline & no & $34(14,5 \%)$ & $9(8,4 \%)^{*}$ & $25(19,6 \%)^{*}$ \\
\hline & $\mathrm{ns} / \mathrm{nc}$ & $37(15,8 \%)$ & $9(8,4 \%)^{*}$ & $28(22,0 \%)^{*}$ \\
\hline \multirow[t]{3}{*}{$\begin{array}{l}\text { Capaz de ponerlo } \\
\text { correctamente }\end{array}$} & si & $145(62,2 \%)$ & $88(83,0 \%)^{*}$ & $57(44,8 \%)^{*}$ \\
\hline & no & $44(18,8 \%)$ & $9(8,4 \%)^{*}$ & $35(27,5 \%)^{*}$ \\
\hline & $\mathrm{ns} / \mathrm{nc}$ & $44(18,8 \%)$ & $9(8,4 \%)^{*}$ & $35(27,5 \%)^{*}$ \\
\hline \multirow[t]{3}{*}{$\begin{array}{l}\text { Rechazaría relación sin } \\
\text { protección }\end{array}$} & si & $150(63,8 \%)$ & $52(48,6 \%)^{*}$ & $98(76,5 \%)^{*}$ \\
\hline & no & $25(10,6 \%)$ & $16(14,9 \%)^{*}$ & $9(7,0 \%)^{*}$ \\
\hline & $\mathrm{ns} / \mathrm{nc}$ & $60(25,5 \%)$ & $39(36,4 \%)^{*}$ & $21(16,4 \%)^{*}$ \\
\hline
\end{tabular}

$* \mathrm{p}<0,05$

Para el análisis estadístico ha habido que agrupar en ocasiones dos clases (No y ns/nc), empleándose entonces la ji con corrección de Yates o la probabilidad exacta de Fischer.

A los seis meses de la intervención respondieron al segundo cuestionario 197 adolescentes (edad media 15,83; 56,56\% mujeres). Había participado en el concurso el $49,48 \%$, y de ellos les había parecido útil o muy útil al 88,57\%.

En lo referente a conocimientos y actitudes sobre el uso del preservativo (tabla 3 ), en el $2^{\circ}$ test se encontró mejoría significativa en el conocimiento de la capacidad preventiva frente al sida-ETS (del $95,76 \%$ pasa a $99,49 \%$; $\mathrm{p}=0,032$ ) y en el uso correcto del preservativo $(62,13 \%$ pasa a $73,46 \% ; \mathrm{p}=0,032)$.

Respecto al sida (tabla 4), se observó mejoría estadísticamente significativa en cuanto al conocimiento de la no transmisión 
Tabla 2

Conocimientos y actitudes sobre sida, por sexos

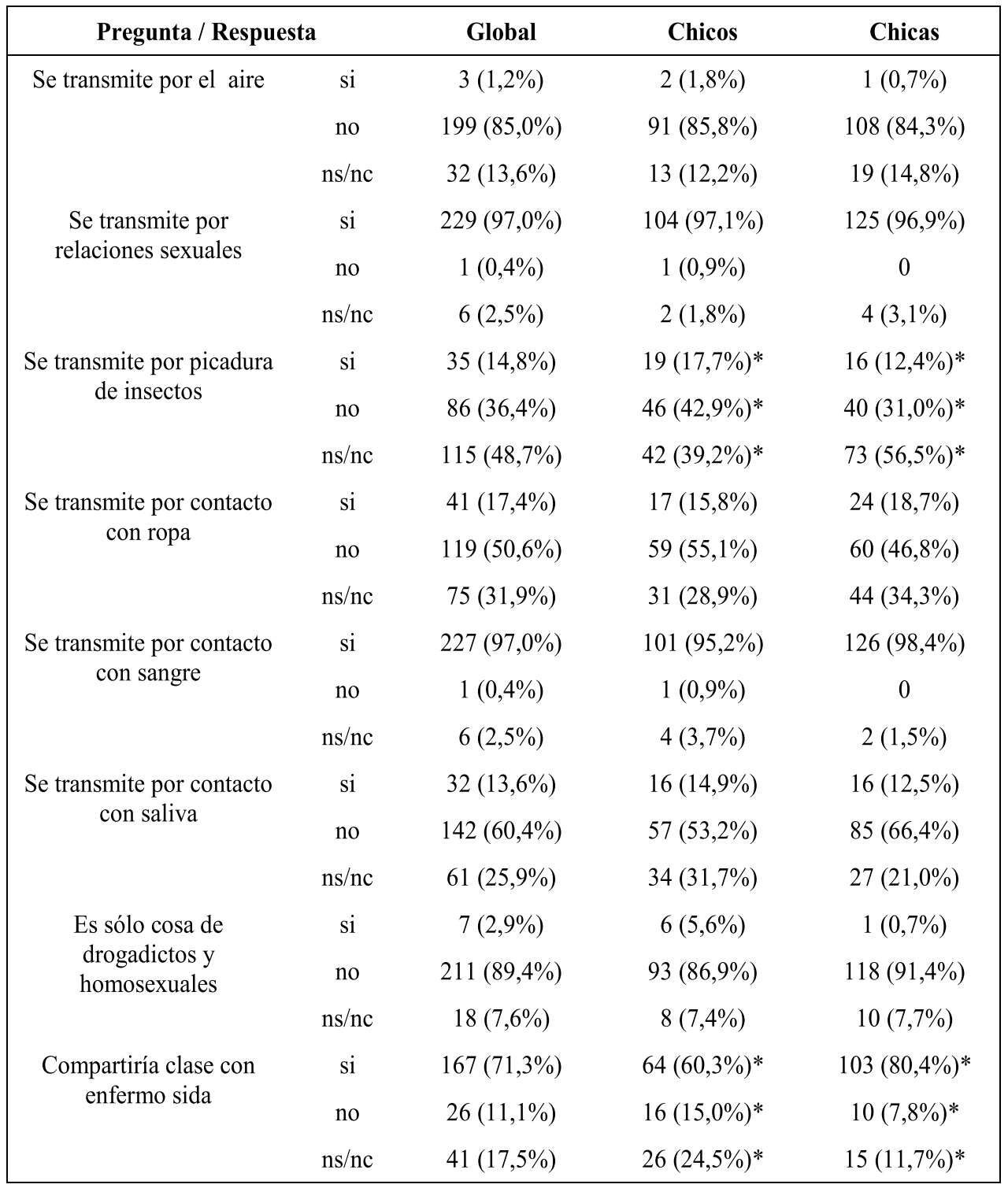

$* \mathrm{p}<0,05$

Nota: Para el análisis estadístico ha habido que agrupar en ocasiones las dos clases de menor tamaño, empleándose entonces la ji con corrección de Yates o la probabilidad exacta de Fischer.

por aire $(\mathrm{p}=0,029)$, por ropa $(\mathrm{p}=0,010)$ y por saliva ( $\mathrm{p}=0,003)$, y casi significativa en que el sida no es exclusivo de homosexuales y drogadictos $(\mathrm{p}=0,09)$. 
Tabla 3

Conocimientos y actitudes sobre preservativo (antes-después)

\begin{tabular}{|lcccc|}
\hline & \multicolumn{2}{c}{$\begin{array}{c}\text { Antes de la intervención } \\
\text { (N= 238) }\end{array}$} & \multicolumn{2}{c|}{$\begin{array}{c}\text { Después de la intervención } \\
\text { (N= 197) }\end{array}$} \\
Rechazaría sin protección & $\mathbf{n}$ & $\mathbf{\%}$ & $\mathbf{n}$ & $\%$ \\
Sé ponerlo correctamente & 152 & 63,8 & 130 & 65,9 \\
Soy capaz de comprarlo & 146 & $61,3^{*}$ & 144 & $73,0^{*}$ \\
No reduce placer & 164 & 68,9 & 139 & 70,5 \\
Previene embarazo & 73 & $30,6^{*}$ & 86 & $43,6^{*}$ \\
Previene ETS y sida & 230 & 96,6 & 190 & 96,4 \\
\hline
\end{tabular}

$* \mathrm{p}<0,05$

Tabla 4

Conocimientos y actitudes sobre sida (antes-después)

\begin{tabular}{|l|c|c|c|c|}
\hline & \multicolumn{2}{|c|}{$\begin{array}{c}\text { Antes de la intervención } \\
\text { (N=238) }\end{array}$} & \multicolumn{2}{c|}{$\begin{array}{c}\text { Después de la intervención } \\
\text { (N=197) }\end{array}$} \\
\hline & $\mathbf{n}$ & $\%$ & $\mathbf{n}$ & $\%$ \\
\hline No es sólo de homosexuales & 213 & 89,4 & 185 & 93,9 \\
\hline Compartiría clase con alumno VIH & 169 & 71,0 & 153 & 77,6 \\
\hline Transmisión por sangre & 228 & 95,7 & 193 & 97,9 \\
\hline No por compartir ropa* & 121 & 50,8 & 125 & 63,4 \\
\hline No transmisión por insectos & 88 & 36,9 & 80 & 40,6 \\
\hline Transmisión sexual & 231 & 97,0 & 193 & 97,9 \\
\hline No transmisión por aire* & 201 & 84,4 & 181 & 91,8 \\
\hline No transmisión por saliva* & 143 & 60,0 & 144 & 73,0 \\
\hline
\end{tabular}

$* \mathrm{p}<0,05$

\section{DISCUSIÓN}

La intervención fue ofertada a todos los centros educativos con alumnos de $4^{\circ}$ de ESO de la ciudad. Los cinco centros que aceptaron participar fueron dos privados y tres públicos, proporción similar a la de la totalidad de los centros de Toledo (6/8), por lo que pueden ser considerados representati- vos. A pesar de ello, siempre cabe la posibilidad de un sesgo por la baja participación, aunque las razones aducidas por los no participantes eran de carácter organizativo.

En el 88,57\% encontramos un porcentaje de adolescentes que han mantenido relaciones sexuales completas superior al de otros estudios (entre el 13 y el 18\% 5,7,18). El que el 
porcentaje sea mayor entre las mujeres es un hecho contrastado en otros estudios ${ }^{5,6,18}$ y traduce un inicio de las relaciones más precoz en las chicas. Respecto a la frecuencia de las relaciones los chicos a estas edades las tienen en menor número, siendo únicas $u$ ocasionales, mientras las chicas las tienen más frecuentemente ${ }^{5,19}$.

La mayoría de los adolescentes utilizan el preservativo como método anticonceptivo, en consonancia con otros estudios ${ }^{5,6}$. Pero aún existe un $12 \%$ de nuestros jóvenes que utilizan «la marcha atrás» y se arriesgan a quedarse o a dejar a la pareja embarazada. También el porcentaje de usuarias de la anticoncepción de emergencia es elevado para estas edades. Ambos hechos son razones suficientes para no bajar la guardia en la prevención de embarazos no deseados ${ }^{19}$.

Respecto a los conocimientos que tienen sobre el preservativo, coinciden, en general, con el del resto de los estudios consulta$\operatorname{dos}^{5,6}$. La mayoría sabe que previenen las ETS/sida, pero son las mujeres las más concienciadas para evitar los embarazos no deseados y las más predispuestas a rechazar una relación sin preservativo.

Es preocupante que uno de cada cuatro jóvenes encuestados no tenga clara su decisión ante la oferta de una relación sin preservativo. Tal vez pueda ser explicado en parte por el hecho de que muchos de ellos no saben usarlo correctamente (más de un tercio según nuestra encuesta). La conclusión es obvia: hay que enseñarles a utilizarlo.

Los adolescentes del estudio conocen que el sida se contagia por las relaciones sexuales y por vía parenteral, pero no tienen claro que el contacto con un enfermo de sida, sin intercambio de fluidos, no transmite la enfermedad o que la saliva no es una fuente de contagio. De hecho, casi la mitad pensaban que podía transmitirse por insectos, quizás confundidos por ciertos mensajes publicitarios televisivos o por desconocer lo que es la transmisión sanguínea, como sugiere un trabajo ${ }^{10}$. Tal vez esta falta de información tenga algo que ver con que una parte importante de los jóvenes mayor que en los estudios consultados ${ }^{10,20}$, tenga inconveniente en compartir clase con un sujeto infectado por VIH. Esta actitud, más frecuente entre los varones, debe ser motivo de reflexión en nuestra sociedad.

Respecto a los resultados de la intervención, al tratarse de un estudio antes-después sin grupo control, los cambios observados han de interpretarse con mucha cautela, ya que no hay ninguna evidencia de que se deban exclusivamente a la intervención realizada.

Por otra parte, para valorar adecuadamente la efectividad de la intervención hemos de tener en cuenta que menos de la mitad de las personas que formaban la muestra se involucró realmente en la intervención. Los resultados en este colectivo son mejores pero hemos creído más razonable presentar los datos globales. Los resultados son modestos pero reales: se observó una mejoría para rechazar relaciones sin protección, aumentó la capacidad para comprar y ponerse el preservativo, mejoraron los conocimientos sobre la transmisión del sida e, incluso, se apunta una mejor actitud para compartir clase con alumnos infectados por VIH.

Creemos que la intervención podría mejorarse si se iniciara a edades más tempranas, cuando los jóvenes no han tenido contacto con el sexo ${ }^{15}$; así, no tendrían que ir encaminadas tanto a cambiar actitudes como a mejorar conocimientos ${ }^{11,12,21}$. No debemos tener miedo a mostrar a los adolescentes cómo debe colocarse un preservativo y debemos hacerlo de la forma más natural posible 22 .

Estamos convencidos también de que los profesionales de atención primaria hemos de involucrarnos en la educación de los adolescentes. Según algunos autores ${ }^{1,17}$, ellos mis- 
mos prefieren recibir información de sus médicos, aunque en la mayoría no son los más accesibles para hablar de sexualidad. Además, para que estas intervenciones tengan los resultados que todos deseamos, es necesario que se realicen en el medio en el que el adolescente se desenvuelve habitualmente con mayor libertad, como es su centro de enseñanza ${ }^{23,24}$.

No basta con aportar información de forma rutinaria; ésta ha de ser verosímil y atractiva a la vez, para que los adolescentes, que se encuentran en una época difícil de su vida, sepan valorarla adecuadamente ${ }^{7}$. Podemos ir más allá aún, e intentar que sean los propios adolescentes, informados y asesorados por nosotros y sus profesores, los que transmitan a sus compañeros sus conocimientos $^{12}$.

\section{BIBLIOGRAFÍA}

1. Romero RJ, Lora MN, Cañete R. Adolescentes y fuentes de información de sexualidad: preferencias y utilidad percibida. Aten Primaria. 2001; 27 : 12-7.

2. Pensemos en este momento: lo que los adolescentes quieren que otros adolescentes sepan sobre la prevención del embarazo. The Nacional Campaign To Prevent Teen Pregnancy. Washington, DC; 2002. Disponible en: www.teenpregnancy.org/ resources/reading/hispanic/espanol4.asp [consultado el 14-8-03].

3. En Anticoncepción de Emergencia infórmate ya en tu web. Dreiman farma. Alcalá de Henares; 2003. Disponible en:: www.informateya.com [consultado el 14-8-03].

4. Tu vida no es un juego. Practica sexo seguro. Utiliza preservativo. Campaña contra el sida. Junta de Comunidades de Castilla-La Mancha. Toledo, 2003. Disponible en: www.jccm.es [consultado el 25-9-03].

5. Informe Juventud en España 2000. Instituto de la Juventud. Disponible en: www.mtas.es/injuve/ index.htm [consultado el 6-9-03].

6. Vergara JC, López-Guerrero A, López F. Anticoncepción de emergencia: perfil de la usuaria en ser- vicios de urgencias de atención primaria. Aten Primaria. 2004; 34(6): 279-85.

7. Ras E, Lluis M, Subirats R, Pellejo ML, Lara A, Rodríguez MV. La educación sexual en los adolescentes desde la atención primaria. Salud Rural 2004; XXI (3): 89-96.

8. Kirby DB, Baumler E, Coyle KK, Basen-Engquist K, Parcel GS, Harrist R, Banspach SW. The «Safer Choices» intervention: its impact on the sexual behaviors of different subgroups of high school students. J Adolesc Health 2004; 35(6):442-52.

9. Robin L, Dittus P, Whitaker D, Crosby R, Ethier K, Mezoff $\mathrm{J}$ et al. Behavioral interventions to reduce incidence of HIV, STD, and pregnancy among adolescents: a decade in review. J Adolesc Health 2004; 34(1):3-26.

10. Rebull J, Reverté M, Piñas I, Ortí A, González L, Contreras E. Evaluación pre-post de una actividad preventiva de la infección por VIH dirigida a adolescentes de las comarcas del sur de Tarragona. Rev Esp Salud Pública. 2003; 77: 373-82.

11. Juárez O, Díez E. Prevención del sida en adolescentes escolarizados: una revisión sistemática de la efectividad de las intervenciones. Gac Sanit 1999; 13 (2): 150-62.

12. Fernández S, Juárez O, Díaz E. Prevención del sida en la escuela secundaria: recopilación y valoración de programas. Rev Esp Salud Pública 1999; 73 : 687-96.

13. Rodríguez MA. Embarazos no deseados: visión general de la situación. Jornadas «Jóvenes y sexualidad: algunas situaciones de exclusión». Santander 2000. Disponible en: www.cje.org/publicaciones. nsf/docs/599JF9ISAZ? opendocument [consultado el 19-5-04].

14. Muñoz E, Mattos I, Peramo B. Anticoncepción y sexualidad en la adolescencia. Tokoginecol Pract. 1999; 58 (635): 81-7.

15. Consejos para evitar el embarazo en adolescentes. The Nacional Campaign To Prevent Teen Pregnancy. Washington, DC 2002. Disponible en: www.teenpregnancy.org/resources/reading/hispanic/espanol4.asp [consultado el 14-8-03].

16. Hernán M, Ramos M, Fernández A. Salud y Juventud. Escuela Andaluza de Salud Pública. Ed. Consejo de la Juventud de España. Madrid. 2002. Disponible en: www.cje.org/publicaciones.nfs/ docs/5EWCZ91SAZ! opendocument [consultado el 7-9-03] 
17. Gascón JA, Navarro B, Gascón FJ, Pérula LA, Jurado A, Montes G. Sexualidad y fuentes de información en población escolar adolescente. Medicina de Familia (And). 2003; 4 (2): 124-9.

18. Juárez O, Díez E, Barniol J, Villamaría F, Nebot M, Villalba JR. Conductas preventivas de la transmisión sexual de sida, de otras infecciones y del embarazo en estudiantes de secundaria. Aten Primaria 1999; 24 (4): 194-202.

19. Parera N, Suris JC. Sexuality and contraception in adolescents from Barcelona, Spain. J Pediatr Adolesc Gynecol 1997; 10; 153-7.

20. González J, Calvo JR, Prats P. Estudio epidemiológico de comportamientos de riesgo en adolescentes escolarizados de dos poblaciones, semirural y urbana. Aten Primaria. 2002; 30 (4): 214-9.
21. Díez E, Barniol J, Nebot M, Juárez O, Martín M, Villalbí JR. Comportamientos relacionados con la salud en estudiantes de secundaria: relaciones sexuales y consumo de tabaco, alcohol y cannabis. Gac Sanit. 1998; 12 (6): 272-80.

22. Mesa MI, Varella JL, Cobeña M. Comportamientos sexuales y uso de preservativos en adolescentes de nuestro entorno. Aten Primaria. 2004; 33(7): 374-80.

23. Los jóvenes responden: diez cosas que desean los adultos sepan sobre el embarazo en la adolescencia. The Nacional Campaign To Prevent Teen Pregnancy. Washington, DC 2002. Disponible en: [consultado el 14-8-03].

24. Romero de Castilla RJ, Lora MN, Cañete R. Adolescentes y fuentes de información de sexualidad: preferencias y utilidad percibida. Aten Primaria. 2001; 27: 12-7. 